DOI: 10.15690/vsp.v18i3.2034

О.В. Солонина ${ }^{1}$, Т.М. Сы ${ }^{2}$

1 Южно-Сахалинская детская городская поликлиника, Южно-Сахалинск, Российская Федерация

2 Областная детская больница, Южно-Сахалинск, Российская Федерация

\title{
Безопасность четырехвалентной
} конъюгированной менингококковой вакцины у детей двухлетнего возраста: проспективное когортное исследование в Сахалинской области

Контактная информация:

Солонина Оксана Викторовна, врач-инфекционист Южно-Сахалинской детской городской поликлиники

Адрес: 693023, Южно-Сахалинск, ул. Емельянова, д. 2, тел.: +7 (424) 251-04-01, e-mail: oksana64-64@mail.ru

Статья поступила: 30.12.2018 г., принята к печати: 26.06.2019 г.

Обоснование. Переносимость российскими детьми рутинной иммунизации вакциной к менингококкам серогрупп А, C, W, Y ранее не изучалась. Цель исследования - изучить безопасность применения поливалентной конъюгированной менингококковой вакцины у детей двухлетнего возраста в условиях реальной клинической практики. Методы. С апреля 2018 г. на территории Сахалинской области осуществлялась вакцинация детей в возрасте 2 лет против менингококковой инфекции. В исследование включали всех детей, получивших однократно конъюгированную четырехвалентную вакцину к менингококкам серогрупп A, C, W, Y в период с апреля по ноябрь 2018 г. Безопасность вакцинации в общей группе оценивали по обращаемости к участковым педиатрам в связи с общими и местными нежелательными явлениями на введение вакцины в течение 1 мес после вакцинации. Изучение безопасности вакцинации путем опроса родителей выполнено для детей, привитых в период с сентября по октябрь 2018 г. Результаты. В период исследования было вакцинировано 1250 детей. В течение 1 мес после вакцинации участковыми педиатрами было зафиксировано 11 (0,9\%) обращений по причине нежелательных реакций, все - легкой степени: инфильтраты в месте введения вакцины (у 4), повышение температуры до $38^{\circ} \mathrm{C}$ (у 7). Опрос 100 родителей показал, что хотя бы одно побочное явление в течение 4 нед после вакцинации наблюдалось у 23 детей, из них местные реакции (уплотнение, покраснение в месте инъекции) - у 14, общие реакции (непродолжительное повышение температуры до $\left.38,7^{\circ} \mathrm{C}\right)-$ у 9 . Нежелательные явления не потребовали врачебного вмешательства. Заключение. После иммунизации детей двухлетнего возраста конъюгированной четырехвалентной вакциной к менингококкам серогрупп A, C, W, Y нежелательные реакции отмечены менее чем в 1\% случаев по обращаемости к участковым педиатрам, и в каждом четвертом - по результату опроса родителей. Ключевые слова: менингококковая инфекция, вакцинация, конъюгированная менингококковая вакцина, дети, рутинная иммунизация, безопасность, нежелательные явления.

(Для цитирования: Солонина О.В., Сы Т.М. Безопасность четырехвалентной конъюгированной менингококковой вакцины у детей двухлетнего возраста: проспективное когортное исследование в Сахалинской области. Вопросы современной педиатрии. 2019; 18 (3): 175-179. doi: 10.15690/vsp.v18i3.2034)

\section{ОБОСНОВАНИЕ}

Менингококковая инфекция является инфекционным заболеванием с острым началом, быстрым развитием симптомов и тяжелым течением, часто приводящим к летальному исходу. Инвазивные формы менингококковой инфекции требуют срочной госпитализации в отделение интенсивной терапии [1]. Однако даже в случае своевременной госпитализации и проведения всех необходимых лечебно-диагностических мероприятий риск летального исхода сохраняется. В России, например, летальность при инвазивной менингококковой инфекции достигает 18\% [2]. Почти у 30\% пациентов, выживших после инвазивной менингококковой инфекции, отмечаются тяжелые инвалидизирующие осложнения [3]. Наиболее восприимчивыми к менингококковой инфекции являются дети в возрасте до 5 лет, у которых показатель заболеваемости может в десятки раз превышать таковой у взрослых [2]. В Сахалинской области заболеваемость генерализованными формами менингококковой инфекции в 2017 г. увеличилась вдвое по сравнению с предыдущим годом и составила 0,82 на 100 тыс. населения, что на $75 \%$ превышает среднероссийский показатель (0,47 на 100 тыс. населения). При этом все случаи инфекции были зафиксированы у детей в возрасте до 14 лет [4].

Вакцинация является эффективным методом профилактики менингококковой инфекции. В большинстве стран мира вакцинация детей от менингококковой инфекции включена в национальные программы 
иммунизации [5]. В ходе клинического исследования в России и Индии были продемонстрированы иммуногенность и безопасность четырехвалентной конъюгированной вакцины при ее двукратном введении детям в возрасте от 9 до 17 мес жизни [6]. До настоящего времени исследования широкого внедрения вакцинации от менингококковой инфекции в России не проводились, а иммунопрофилактика осуществляется главным образом в эпидемиологических очагах либо по желанию родителей.

Цель исследования - изучить безопасность применения поливалентной конъюгированной менингококковой вакцины у детей двухлетнего возраста в условиях реальной клинической практики.

\section{МЕТОДЫ}

\section{Дизайн исследования}

В связи с неблагоприятной эпидемической ситуацией на территории Сахалинской области с апреля 2018 г. осуществлялась вакцинация против менингококковой инфекции детей двухлетнего возраста. Параллельно с этим нами проведено проспективное когортное исследование безопасности вакцинации. Включение в исследование продолжалось до 31 октября 2018 г.

\section{Условия проведения исследования}

Исследование выполнено на базе детской городской поликлиники № 2 г. Южно-Сахалинска.

\section{Критерии соответствия}

В исследование включали всех детей в возрасте 2 лет (от 24 до $36 \mathrm{мес),} \mathrm{прикрепленных} \mathrm{к} \mathrm{детской} \mathrm{поликлинике,}$ вакцинированных однократно поливалентной конъюги- рованной менингококковой вакциной в период исследования (с апреля по октябрь 2018 г.).

\section{Вакцинация}

Для вакцинации детей использовали вакцину конъюгированную четырехвалентную к менингококкам серогрупп A, C, W, Y (Санофи Пастер, США). Вакцинация детей осуществлялась в прививочном кабинете детской поликлиники при отсутствии временных или постоянных противопоказаний к применению вакцинного препарата. В соответствии с инструкцией к препарату вакцина вводилась однократно в дозе 0,5 мл в дельтовидную мышцу. Допускалось совместное применение менингококковой вакцины с другими вакцинами, назначенными лечащим врачом, при их введении в другое плечо, но не более двух вакцин одновременно.

\section{Оценка безопасности вакцинации}

Безопасность вакцинации в общей группе оценивали по обращаемости к участковым педиатрам в связи с общими и местными нежелательными побочными реакциями (НПР) на введение вакцины в течение 1 мес после вакцинации. НПР считали любые нарушения в состоянии здоровья, которые могли иметь связь с вакцинацией. Все родители были инструктированы сообщать о любых проявлениях в поствакцинальном периоде. Данные о зарегистрированных НПР в течение всего периода исследования (май-октябрь 2018 г.) участковые педиатры сообщали исследователю (О.В. Солонина).

В зависимости от выраженности НПР относили к легким, средней степени выраженности или тяжелым. К легким местным НПР относились инфильтраты и покраснения в месте инъекции диаметром не более 5 см,

Oxana V. Solonina ${ }^{1}$, Tatiana M. Sy ${ }^{2}$

${ }^{1}$ Yuzhno-Sakhalinsk Children's Outpatients Clinic, Yuzhno-Sakhalinsk, Russian Federation

${ }^{2}$ Regional Children's Hospital, Yuzhno-Sakhalinsk, Russian Federation

\section{The Safety of 4-valent Meningococcal Conjugate Vaccine in Two Years Old Children: Prospective Cohort Study in Sakhalin Region}

Background. Acceptability of routine immunization with vaccine to meningococcus serogroups A, C, W, Y in Russian children have not been studied earlier. Objective. The aim of the study was to understand the safety of polyvalent meningococcal conjugate vaccine administration in two years old children in actual clinical practice. Methods. The vaccination of two years old children against meningococcal disease has been performing in Sakhalin region since April 2018. All children who received single 4-valent meningococcal conjugate vaccine to $A, C, W, Y$ serogroups in the period from April to November 2018 were included into the research. The vaccine safety in general group was estimated by local pediatricians visiting due to general and local adverse effects on vaccine injection in one month period. Safety study for children vaccinated in the period from September to October 2018 was carried out by questioning parents. Results. 1250 children were vaccinated during this research. Local paediatricians has recorded 11 (0.9\%) appointments due to adverse effects within 1 month after vaccination. All of them were mild: infiltrates in the vaccine injection site (4 cases), high temperature up to $38^{\circ} \mathrm{C}$ ( 7 cases). Survey of 100 parents has shown that at least one adverse effect in 4 weeks period was noted only in 23 children: local reactions (induration, redness at the injection site) in 14 cases, general reactions (temperature increase up to $38.7^{\circ} \mathrm{C}$ ) in 9 cases. No adverse effects needed medical intervention. Conclusion. Adverse effects were recorded in less than $1 \%$ of all cases according to local paediatricians appointments and in every 4 th case according to parents questioning among all two years old children who undergone immunization with 4-valent meningococcal conjugate vaccine to $A, C, W, Y$ serogroups.

Key words: meningococcal disease, vaccination, meningococcal conjugate vaccine, children, routine immunization, safety, adverse effects.

(For citation: Solonina Oxana V., Sy Tatiana M. The Safety of 4-valent Meningococcal Conjugate Vaccine in Two Years Old Children: Prospective Cohort Study in Sakhalin Region. Voprosy sovremennoi pediatrii - Current Pediatrics. $2019 ; 18$ (3): $175-179$. doi: $10.15690 / v s p . v 18 i 3.2034)$ 
сохраняющиеся не более 7 сут после введения вакцины. Среднетяжелой местной реакцией считалось появление инфильтрата диаметром более 5 см, но в пределах 10 см, с повышением местной температуры, проходящее самостоятельно. Тяжелые местные реакции - холодные абсцессы в месте введения, инфильтраты более 10 см в диаметре, требующие хирургического вмешательства. К легким общим НПР относились кратковременное повышение температуры тела (выше $37,3^{\circ} \mathrm{C}$, но не более $38^{\circ} \mathrm{C}$ ), ощущение слабости, нарушение сна, головная боль, расстройство стула, проходящие самостоятельно. К среднетяжелым общим НПР относились любые состояния, требующие госпитализации ребенка, к тяжелым - анафилаксия или любое другое жизнеугрожающее состояние, развившееся в ответ на введение вакцины.

Детальное изучение безопасности вакцинации выполнено путем опроса первых 100 родителей детей, вакцинированных в период с сентября 2018 г., согласившихся фиксировать в формализованном опроснике НПР в течение 4 нед после вакцинации. В опросниках были предварительно перечислены наиболее распространенные общие (повышение температуры тела $>37,3^{\circ} \mathrm{C}$, рвота, необычный плач, сонливость, потеря аппетита, раздражительность) и местные НПР (уплотнение, покраснение, припухлость). О неожидаемых проявлениях (не указанных в опроснике) в поствакцинальном периоде родители должны были сообщать участковому педиатру. В случае выявления НПР дети осматривались врачом в течение суток после возникновения события с целью оценки предполагаемой связи с вакцинацией.

\section{Анамнестическая характеристика вакцинированных}

Для изучения соматического и вакцинального статуса анализировали медицинскую документацию (форма 112) первых 300 детей, вакцинированных в период с мая 2018 г. Изучали анамнез жизни детей, наличие сопутствующей патологии (аллергоанамнез, фоновые заболевания, прививочный календарь). Результаты обследования этой группы экстраполировали на общую выборку вакцинированных в период исследования.

\section{Этическая экспертиза}

У родителей получали устное согласие на участие детей в исследовании. Согласия Этического комитета на проведение исследования получено не было, протокол исследования в Этический комитет не подавался. Вакцинация осуществлялась в связи с реализацией региональной программы защиты детей от менингококковой инфекции, в которую включали всех детей, достигших двухлетнего возраста.

\section{Статистический анализ}

Необходимый размер выборки предварительно не определяли. Описание количественных переменных выполнено с указанием среднего арифметического значения (стандартное отклонение).

\section{РЕЗУЛЬТАТЫ}

\section{Участники исследования}

В исследование были включены 1250 вакцинированных детей (девочек 53,5\%), средний возраст 27 мес (2 года 3 мес). у 300 детей, случайно выбранных из 1250, были проанализированы амбулаторные карты (наличие фоновых и сопутствующих заболеваний). Так, 42 (14\%) ребенка имели фоновые заболевания: у 12 (4\%) диагностирован атопический дерматит, у 16 (5\%) - различные неврологические нарушения, у 5 (2\%) - нарушения со стороны желудочно-кишечного тракта (у 4 детей - дисбактериоз). Недоношенными (гестационный возраст при рождении 28-32 нед) были 9 (3\%) вакцинированных. У 27 (9\%) детей отсутствовали $\geqslant 1$ обязательных по возрасту профилактических прививок в связи с медицинскими противопоказаниями. В анамнезе у 8 (3\%) детей имелись указания на лекарственную аллергию к антибиотикам (цефотаксим, флемоксин, азитромицин). Указания на непереносимость вакцин отсутствовали.

\section{Основные результаты исследования}

В общей группе вакцинированных зарегистрировано 11 (0,9\%) обращений за медицинской помощью по поводу НПР, при этом среди всех зарегистрированных обращений выявлялись только реакции легкой степени. У 4 детей были зарегистрированы местные проявления (инфильтраты размером не более 1,5 см), послужившие поводом для обращения к лечащему врачу. У 2 детей из 4 отмечалась умеренная болезненность инфильтратов при пальпации. Местные реакции прошли самостоятельно на 2-3-и сут. Повышение температуры от 37,4 до $38^{\circ} \mathrm{C}$ было у 7 детей. Температура повышалась в течение 24 ч после инъекции, прошла самостоятельно через 2 ч у 3 детей; 4 ребенка получили однократно дозу жаропонижающего средства (ибупрофен).

Одновременно с поливалентной конъюгированной менингококковой вакциной в соответствии с индивидуальным графиком прививок были вакцинированы 308 (24\%) детей, из них 43 против краснухи, 160 против гриппа, 17 против вируса гепатита A, 35 против полиомиелита (инактивированная полиомиелитная вакцина у 15, оральная полиомиелитная у 20 детей), против пневмококковой инфекции (конъюгированная вакцина) - 15 детей и одновременно с АКДС - 38. Из 11 детей, обратившихся по поводу НПР, 6 были одновременно привиты против гриппа, у всех отмечалась общая НПР в виде кратковременного повышения температуры тела.

Согласно опросу родителей (группа была сопоставима по полу и возрасту с 1150 детьми, родители которых не участвовали в опросе), в течение 4 нед после вакцинации хотя бы одна НПР была зафиксирована у 25 детей, из них у 14 - местные (уплотнение у 9, покраснение у 2, припухлость у 3), у 11 - общие НПР (в 9 случаях повышение температуры тела выше $37,3^{\circ} \mathrm{C}$, но не более 38,8 ; у 1 ребенка развилась респираторная инфекция, у 1 ребенка - назофарингит). Большинство НПР (23 из 25) были ожидаемыми, все проходили самостоятельно и не требовали дополнительной терапии. Общие реакции в виде лихорадки 
у 7 из 9 детей возникли после одновременного применения менингококковой и трехвалентной инактивированной противогриппозной вакцины.

\section{ОБСУЖДЕНИЕ}

\section{Резюме основного результата исследования}

В общей выборке детей отмечалась низкая (менее 1\%) частота обращений в связи с НПР в поствакцинальном периоде. В связи с этим можно констатировать хорошую переносимость конъюгированной менингококковой вакцины, в т.ч. и ввиду отсутствия среднетяжелых или тяжелых проявлений. Следует, однако, учитывать, что при опросе родителей было обнаружено, что доля вакцинированных детей с НПР была заметно выше. Тем не менее все зарегистрированные НПР были легкими и проходили, как правило, самостоятельно.

\section{Ограничения исследования}

Ограничениями исследования, прежде всего, являются отсутствие группы сравнения и наблюдательный дизайн исследования. Также следует учитывать, что в исследование были включены дети, не имевшие противопоказаний к вакцинации. По данным регионального отделения Росстата, в Южно-Сахалинске в 2018 г. проживало 1476 детей в возрасте 2 лет, т.е. невакцинированными оставались чуть более $15 \%$ детей данной возрастной группы. Причины неполного охвата вакцинацией в настоящем исследовании не изучались. Не исключено, что часть детей могла быть вакцинирована после стабилизации состояния по соматическому заболеванию или в условиях стационара под наблюдением врача. Тем не менее однозначно судить о профиле безопасности конъюгированной менингококковой вакцины у этой категории целевой популяции невозможно.

Поскольку исследование проводилось в условиях реальной клинической практики, не все родители детей общей выборки вакцинированных ( $n=1250)$ получили опросник для фиксации НПР. Данный факт мог предопределить относительно небольшую в сравнении с данными регистрационных исследований конъюгированной менингококковой вакцины [7-9] частоту НПР, отмеченных в общей выборке исследования. Вместе с тем опрошенные родители ( $n=100)$ могли неправильно интерпретировать НПР, в частности некоторые местные или слабовыраженные общие реакции не расценивать как повод обращения к врачу, что также могло занизить наблюдаемую частоту НПР.

Следует учитывать, что при оценке соматического статуса использовали сведения, полученные из амбулаторных карт. По этой причине (отсутствие достоверных сведений в медицинской документации) не исключено, что представлены неточные данные о распределении вакцинированных детей по состоянию здоровья (только 14\% с указанием на различные заболевания и патологические состояния).

\section{Интерпретация результатов исследования}

Хорошая переносимость четырехвалентной конъюгированной менингококковой вакцины у детей с различны- ми отклонениями в состоянии здоровья уже отмечалась ранее [8, 10, 11]. При этом частота НПР, зарегистрированная в настоящем исследовании, не превышала данные регистрационных и постмаркетинговых исследований $[12,13]$. В случае развития общих НПР у детей при одновременном введении менингококковой и противогриппозной вакцин не представлялось возможным дифференцировать вклад каждой из вакцин в развитие данного побочного эффекта. При этом бо́льшая частота НПР у детей, получивших одновременно две вакцины, позволяет предположить ведущую роль в развитии данного НПР противогриппозной вакцины [10, 11]. Традиционно высокие показатели НПР, получаемые в ходе регистрационных исследований вакцины, связаны прежде всего с локальными и слабовыраженными общими реакциями на введение иммунобиологического препарата $[12,13]$. Так, по данным проведенного нами опроса, родители значительно чаще отмечали побочные реакции, характерные для течения поствакцинального периода. При этом необходимо отметить, что данные НПР проходили самостоятельно и не служили поводом для обращения к врачу у подавляющего большинства родителей, не попавших в группу углубленного изучения профиля безопасности.

\section{ЗАКЛЮЧЕНИЕ}

Проанализирована переносимость четырехвалентной конъюгированной менингококковой вакцины у детей в возрасте 2 лет. Частота НПР варьировала от < 1\% (по данным обращаемости к участковым педиатрам) до 25\% (по результатам опроса родителей). При этом все НПР носили легкий характер и, как правило, проходили самостоятельно.

\section{ИСТОЧНИК ФИНАНСИРОВАНИЯ}

Публикация статьи осуществлена при поддержке компании «Санофи Пастер".

\section{FINANCING SOURCE}

Article is published with support of Sanofi Pasteur.

\section{КОНФЛИКТ ИНТЕРЕСОВ}

Авторы статьи подтвердили отсутствие конфликта интересов, о котором необходимо сообщить. Авторы также подтвердили, что сотрудники компании "Санофи Пастер" не имели отношения к планированию, проведению и анализу результатов настоящего исследования.

\section{CONFLICT OF INTERESTS}

Oxana V. Solonina and Tatiana M. Sy confirmed the absence of a reportable conflict of interests. The contributors have also confirmed that Sanofi Pasteur employees were irrelevant to planning, carrying out and outcomes analysis of this research.

\section{ORCID}

\section{О.В. Солонина}

https://orcid.org/0000-0002-6568-9588 


\section{СПИСОК ЛИТЕРАТУРЫ}

1. Менингококковая инфекция у детей (эпидемиология, клиника, диагностика, терапия и профилактика). Методические рекомендации. / Под редакцией Ю.В. Лобзина. - СПб., 2009. 60 c. [Meningokokkovaya infektsiya u detej (epidemiologiya, klinika, diagnostika, terapiya i profilaktika). Metodicheskie rekomendatsii. Ed by Yu.V. Lobzin. St. Petersburg; 2014. 60 p. (In Russ).]

2. Информационно-аналитический обзор "Менингококковая инфекция и гнойные бактериальные менингиты в Российской Федерации, 2017 год". - М.: Российский референс-центр по мониторингу за бактериальными менингитами ЦНИИ эпидемиологии Роспотребнадзора РФ, 2018. [Informatsionno-analiticheskij obzor "Meningokokkovaya infektsiya i gnojnye bakterial'nye meningity v Rossijskoj Federatsii, 2017 god". Moscow: Rossijskij referens-tsentr po monitoringu za bakterial'nymi meningitami TsNII epidemiologii Rospotrebnadzora RF; 2018. (In Russ).]

3. Sadarangani M, Pollard AJ. Can we control all-cause meningococcal disease in Europe? Clin Microbiol Infect. 2016;22 Suppl 5:S103-S112. doi: 10.1016/j.cmi.2016.03.006.

4. Государственный доклад от 14 августа 2018 г. "О состоянии санитарно-эпидемического благополучия населения в Сахалинской области в 2017 году". - Южно-Сахалинск, 2018. [State report "O sostoyanii sanitarno-epidemicheskogo blagopoluchiya naseleniya v Sakhalinskoj oblasti v 2017 godu", dated 2018 August 14. Yuzhno-Sakhalinsk, 2018. (In Russ).] Доступно по: http://65.rospotrebnadzor.ru/documents/regional/ doklad/. Ссылка активна на 25.03.2019.

5. Фельдблюм И.В., Новгородова С.Д., Гореликова Е.В. Эпидемиология и новые возможности специфической профилактики менингококковой инфекции в условиях реального времени // Поликлиника. - 2018. - № 2. - C. 24-27. [Feldblyum IV, Novgorodova SD, Gorelikova EV. Epidemiology and new opportunities for real-time specific prevention of meningococcal infection. Poliklinika. 2018;(2):24-27. (In Russ).]

6. Javadekar B, Ghosh A, Kompithra RZ, et al. Safety and immunogenicity of a two-dose schedule of a quadrivalent meningococcal polysaccharide diphtheria toxoid conjugate vaccine in Russian and Indian children aged 9 to 17 months. Indian Pediatrics. 2018;55(12):1050-1055. doi: 10.1007/s13312018-1440-z.

7. Фридман И.В., Харит С.М. Профилактика менингококковой инфекции // Медицинский совет. - 2017. - № 4 . C. 16-18. [Fridman IV, Kharit SM. Prevention of meningococcal infection. Meditsinskij sovet. 2017;(4):16-18. (In Russ).] doi: 10.21518/2079-701X-2017-4-16-18.

8. Намазова-Баранова Л.С., Новикова Д.А., Федосеенко М.В., и др. Безопасность совместного применения четырехвалентной конъюгированной вакцины против менингококковой инфекции серогрупп A,C,Y,W-135 с другими вакцинными препаратами: проспективное исследование серии случаев среди детей здоровых и с различными отклонениями в состоянии здоровья // Вопросы современной педиатрии. - 2017. - Т. 16. - № 2. - С. 156-162. [Namazova-Baranova LS, Novikova DA, Fedoseenko MV, et al. Safety of combination of a tetravalent meningococcal conjugate vaccine against serogroups $\mathrm{A}, \mathrm{C}, \mathrm{Y}, \mathrm{W}-135$ with other vaccine preparations: a prospective study of a series of cases among healthy children and children with various health abnormalities. Current Pediatrics. 2017;16(2):156-162. (In Russ).] doi: 10.15690/vsp.v16i2.1717.

9. Ртищев А.Ю., Шамшева О.В. Оценка безопасности вакцинации против менингококковой инфекции в период эпидемического подъема заболеваемости в городе Москве. / Материалы III Конгресса педиатров-инфекционистов России "Актуальные вопросы инфекционной патологии у детей. Инфекция и иммунитет»; 8-10 декабря; Москва. - М.: Ассоциация педиатровинфекционистов, 2004. [Rtishchev AYu, Shamsheva OV. Otsenka bezopasnosti vaktsinatsii protiv meningokokkovoj infektsii v period epidemicheskogo pod"ema zabolevaemosti v gorode Moskve. Materialy III Kongressa pediatrov-infektsionistov Rossii “Aktual'nye voprosy infektsionnoj patologii u detej. Infektsiya i immunitet»; dated 2004 Dec 8-10. Moscow: Assotsiatsiya pediatrov-infektsionistov; 2004. (In Russ).]

10. Намазова Л.С., Костинов М.П., Волкова О.Н., и др. Профилактика гриппа, ОРИ, пневмококковой, менингококковой и Нib-инфекции часто болеющих детей: пособие для врачей. M., 2006. - 43 c. [Namazova LS, Kostinov MP, Volkova ON, et al. Profilaktika grippa, ORI, pnevmokokkovoj, meningokokkovoj i Hibinfektsii chasto boleyushchikh detej: posobie dlya vrachej. Moscow; 2006. 43 р. (In Russ).]

11. Вакцинация детей с нарушенным состоянием здоровья: практическое руководство для врачей. 4-е изд. / Под. ред. М.П. Костинова. - М.: Медицина для всех, 2013. - 432 с. [Vaktsinatsiya detej s narushennym sostoyaniem zdorov'ya: prakticheskoe rukovodstvo dlya vrachej. 4th ed. Ed by M.P. Kostinov. Moscow: Meditsina dlya vsekh; 2013. 432 p. (In Russ).]

12. Pina LM, Bassily E, Machmer A, et al. Safety and immunogenicity of a quadrivalent meningococcal polysaccharide diphtheria toxoid conjugate vaccine in infants and toddlers: three multicenter phase III studies. Pediatr Infect Dis J. 2012;31(11):1173-1183. doi: 10.1097/INF.0b013e318268dfe4.

13. Sanofi Pasteur. Menactra, Meningococcal (Groups A,C,Y and W-135) Polysaccharide Diphtheria Toxoid Conjugate Vaccine Solution for Intramuscular Injection. Highlights of prescribing information. [revised April 2018] Initial U.S. Approval: 2005. Available from: https://www.vaccineshoppe.com/image.cfm?doc_ id=12580\&image_type=product_pdf. 